\title{
In vitro evaluation of 5-aminolevulinic acid (ALA) loaded PLGA nanoparticles
}

\author{
This article was published in the following Dove Press journal: \\ International Journal of Nanomedicine \\ 23 July 2013 \\ Number of times this article has been viewed
}

\author{
Lei $\mathrm{Shi}^{1}$ \\ Xiuli Wang' \\ Feng Zhao ${ }^{2}$ \\ Hansen Luan ${ }^{2}$ \\ Qingfeng Tu' \\ Zheng Huang ${ }^{3}$ \\ Hao Wang ${ }^{2}$ \\ Hongwei Wang ${ }^{1,4}$ \\ 'Shanghai Skin Disease Hospital, \\ Shanghai, People's Republic of China; \\ ${ }^{2}$ National Pharmaceutical Engineering \\ Research Center, China State Institute \\ of Pharmaceutical Industry, Shanghai, \\ People's Republic of China; ${ }^{3}$ Ministry \\ of Education (MOE) Key Laboratory \\ of OptoElectronic Science and \\ Technology for Medicine, Fujian \\ Normal University, Fuzhou, People's \\ Republic of China; ${ }^{4}$ Huadong Hospital, \\ Fudan University, Shanghai, People's \\ Republic of China
}

Correspondence: Hongwei Wang Shanghai Skin Disease Hospital, I278 Baode Road, Shanghai 200443, People's Republic of China

$\mathrm{Tel}+862161833004$

Fax +86 21 61833021

Email hongweiwang2005@yahoo.com

HaoWang

National Pharmaceutical Engineering Research Center, China State Institute of Pharmaceutical Industry, I 320 Beijing West Road, Shanghai 200040, People's

Republic of China

Tel +86-2I-5I 320728

Fax +86-2I-5I3207/9

Email wanghao99@hotmail.com
Background: 5-Aminolevulinic acid (ALA) is a prodrug for topical photodynamic therapy. The effectiveness of topical ALA can be limited by its bioavailability. The aim of this study was to develop a novel ALA delivery approach using poly(lactic-co-glycolic acid) (PLGA) nanoparticles (NPs).

Methods: A modified double emulsion solvent evaporation method was used to prepare ALA loaded PLGA NPs (ALA PLGA NPs). The characteristics, uptake, protoporphyrin IX fluorescence kinetics, and cytotoxicity of ALA PLGA NPs toward a human skin squamous cell carcinoma cell line were examined.

Results: The mean particle size of spherical ALA PLGA NPs was $65.6 \mathrm{~nm} \pm 26 \mathrm{~nm}$ with a polydispersity index of 0.62 . The encapsulation efficiency was $65.8 \% \pm 7.2 \%$ and ALA loading capacity was $0.62 \% \pm 0.27 \%$. When ALA was dispersed in PLGA NPs, it turned into an amorphous phase. ALA PLGA NPs could be taken up by squamous cell carcinoma cells and localized in the cytoplasm. The protoporphyrin IX fluorescence kinetics and 3-[4,5-dimethylthiazol-2yl]-2,5-diphenyltetrazolium bromide assay showed that ALA PLGA NPs were more effective than free ALA of the same concentration.

Conclusion: PLGA NPs provide a promising ALA delivery strategy for topical ALAphotodynamic therapy of skin squamous cell carcinoma.

Keywords: 5-Aminolevulinic acid (ALA), nanoparticles, poly(lactic-co-glycolic acid) (PLGA), skin squamous cell carcinoma, photodynamic therapy (PDT)

\section{Introduction}

Photodynamic therapy (PDT) is a treatment modality which combines the action of photosensitizer (PS), light, and oxygen. Upon the photoirradiation of PS at specific wavelength(s), photodynamic reactions can generate cytotoxic reactive oxygen species that oxidize subcellular organelles and biomolecules, ultimately leading to the destruction of diseased cells and tissues. ${ }^{1}$

5-Aminolevulinic acid (ALA; molecular weight: 167.6 Da) is a precursor of protoporphyrin IX (PpIX), a potent fluorescent PS. Topical ALA-mediated PDT (ALA-PDT) is based on the accumulation of endogenous PpIX following topical administration of ALA. ${ }^{2}$ ALA-PDT has been successfully used for the management of different types of superficial skin precancer and cancer such as actinic keratosis, basal cell carcinoma, Bowen's disease, and Paget's disease. ${ }^{3-5}$ Compared with surgery, radiation, and chemotherapy, ALA-PDT has the advantages of minimal invasiveness, better aesthetic outcomes, low morbidity, minimal functional disturbance, good tolerance, and the ability to be used repeatedly at the same site. 
Squamous cell carcinoma (SCC) is a common skin cancer, and its treatment is still a challenge. Although ALAPDT shows some promise for the treatment of superficial SCC, ${ }^{6}$ currently, it is not recommended for the treatment of invasive SCC mainly because (1) the local bioavailability of ALA is insufficient for a complete therapeutic effect, despite the fact that ALA can permeate the skin barrier, (2) ALA is unstable in physiological environments, and (3) diffusion of ALA through cell membranes is limited due to its polarity. ${ }^{7,8}$

Several studies suggest that nanoparticle (NP) drug delivery systems (eg, liposomes, chitosan NPs, gold NPs) might be useful for overcoming these deficiencies and enhance the effectiveness of topical ALA-PDT. ${ }^{9-11}$ But these NP systems have some drawbacks such as instability, leakage of encapsulated drug, and unknown long-term side effects. Poly(lacticco-glycolic acid) (PLGA) is a biomaterial developed in the 1970s and approved by the United States Food and Drug Administration (FDA) for drug delivery. ${ }^{12}$ Biodegradable PLGA shows good biocompatibility and stability, therefore making it a better candidate for the delivery of PS..$^{13,14}$ However it has not been tested for encapsulating and delivering ALA. In this study, ALA loaded PLGA nanoparticles (ALA PLGA NPs) were prepared and characterized in an effort to develop a feasible ALA delivery system to improve the efficacy of ALA-PDT for the treatment of skin SCC.

\section{Materials and methods}

\section{Chemicals}

PLGA (lactic acid:glycolic acid ratio of 75:25; molecular weight: $65,921 \mathrm{Da}$ ) was obtained from the National Pharmaceutical Engineering Research Center, China State Institute of Pharmaceutical Industry (Shanghai, People's Republic of China). ALA hydrochloride powder was from Shanghai Fudan-Zhangjiang Bio-Pharmaceutical Co, Ltd (Shanghai, People's Republic of China); acetonitrile, dichloromethane (DCM), and polyvinyl alcohol (Mowiol 5-88) were from SINOPHARM (Beijing, People's Republic of China); fluorescamine was from Alfa Aesar (Ward Hill, MA, USA); and 3-[4,5-dimethylthiazol-2-yl]-2,5diphenyltetrazolium bromide (MTT) was obtained from Sigma-Aldrich (St Louis, MO, USA). All chemicals were of analytical grade.

\section{Quantitative assay of ALA}

ALA concentration was determined after converting ALA into a fluorescent derivative by reacting with fluorescamine [4-phenylspiro (furan-2[3H],1-phthlan)-3,3-dione]

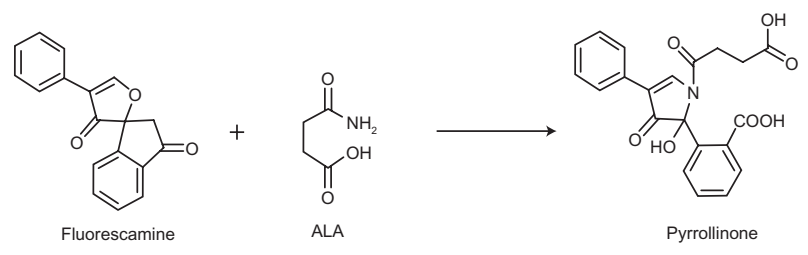

Figure I Reaction of Fluorescamine and ALA (5-aminolevulinic acid).

(Figure 1). The derivative was analyzed by high performance liquid chromatography (HPLC) as described by Namjoshi et al. ${ }^{15}$ In brief, samples and controls were dissolved in phosphate buffered saline (PBS) at pH 5.0. Fluorescamine solution $(0.1 \%)$ was prepared by dissolving fluorescamine in acetone solution. To an HPLC vial, $50 \mu \mathrm{L}$ of sample, $50 \mu \mathrm{L}$ of $0.1 \%$ fluorescamine solution, and $150 \mu \mathrm{L}$ of $0.1 \mathrm{M}$ borate buffer were added and mixed on a vortex mixer. The mixture was allowed to react for 10 minutes at room temperature.

Twenty microliters of the mixture containing the fluorescent derivative was injected into an Inertsil ODS-3 C18 column (GL Sciences, Tokyo, Japan) $(4.6 \mathrm{~mm} \times 250 \mathrm{~mm}$, $5 \mu \mathrm{m})$. The elution was performed at $40^{\circ} \mathrm{C}$ at $1.5 \mathrm{~mL} / \mathrm{min}$. The mobile phase contained $30 \%$ acetonitrile $(0.1 \%$ trifluoroacetic acid [TFA]) and 70\% water (0.1\% TFA). For fluorescence detection, the excitation wavelength was set at $398 \mathrm{~nm}$ and emission at $480 \mathrm{~nm}$ with a bandwidth of $15 \mathrm{~nm}$ for the fluorescence detector (Shimadzu-RF-10 AXL; Shimadzu, Kyoto, Japan). Data were analyzed using the proprietary Shimadzu Class VP software.

\section{Preparation of ALA-loaded PLGA NPs}

ALA PLGA NPs were prepared using a modified double emulsion solvent evaporation technique. ${ }^{16}$ First, PLGA was dissolved in DCM as oil phase and ALA in PBS (pH 5.0) as internal aqueous phase. The aqueous and oil phase were then mixed by sonication on ice with a probe sonicator (YPZD025050; Hangzhou Success Ultrasonic Equipment Co, Ltd, Zhejiang, People's Republic of China) under a discontinuous mode for 40 seconds at 120 Watts. The primary emulsion was transferred into $40 \mathrm{~mL}$ external aqueous phase of $1 \%$ polyvinyl alcohol in PBS (pH 5.0). The mixture was sonicated on ice for another 40 seconds. After emulsification, the water in oil in water emulsion was magnetically stirred for 4 hours to evaporate organic solvent in the colloidal suspension of ALA PLGA NPs in water and isolated by centrifugation at $12,000 \mathrm{rpm}$ at $4^{\circ} \mathrm{C}$ for 15 minutes. NPs were washed three times with distilled water to remove any residual polyvinyl alcohol and unbound ALA. NPs were freeze-dried immediately in the presence 
of $15 \%$ mannitol and stored at $-20^{\circ} \mathrm{C}$. Empty PLGA NPs were prepared without an addition of ALA using the same procedure.

\section{Characterizations of ALA PLGA NPS}

\section{Determination of particle size distribution} and polydispersity

Freeze-dried NPs were resuspended in distilled water and analyzed using a laser diffraction particle size analyzer (LS 230; Beckman Coulter, Brea, CA, USA). The analysis range was $0.04-2000 \mu \mathrm{m}$, real refractive index 1.5 , and imaginary refractive index 0.1 . The polydispersity index (PI) was determined as:

$$
\mathrm{PI}=\frac{\mathrm{D}_{90}-\mathrm{D}_{10}}{\mathrm{D}_{50}}
$$

where $\mathrm{D}_{10}$ corresponds to particle size above $10 \%$ of the sample, $\mathrm{D}_{50}$ to particle size above $50 \%$, and $\mathrm{D}_{90}$ to particle size above $90 \%$.

\section{ALA encapsulation efficiency and loading capacity of nanoparticles}

The encapsulation efficiency (EE) was determined using the colloidal suspension of ALA PLGA NPs in distilled water. Briefly, the suspension was centrifuged at $12,000 \mathrm{rpm}$ at $4^{\circ} \mathrm{C}$ for 15 minutes. The free ALA in the supernatant was removed. The nanoparticles in sediments were collected and dissolved in DCM. ALA was then extracted by the mobile phase and converted to the fluorescent derivative before quantification. The amount of ALA entrapped in nanoparticles was measured as described earlier and the percentage of EE was determined as:

$$
\mathrm{EE} \%=\frac{\text { Amount of ALA entrapped in NPs }}{\text { Initial ALA added }} \times 100 \%
$$

To determine the loading capacity (LC), freeze-dried nanoparticles were weighed and dissolved in DCM. ALA entrapped in nanoparticles was measured as described before. The LC was calculated as:

$$
\mathrm{LC} \%=\frac{\text { Amount of ALA entrapped in NPs }}{\text { Weight of freeze dried NPs }} \times 100 \%
$$

\section{Determination of NP morphology}

ALA PLGA NPs resuspended in ultrapurified water were spread on a sample holder, dried under vacuum, and subsequently coated with a thin layer of colloidal gold before being examined under scanning electron microscopy (XL30FEG; Philips, Eindhoven, The Netherlands).

\section{Determination of physical state of NPs}

Differential scanning calorimetry (DSC) thermograms of pure ALA powder, empty PLGA NPs, ALA and PLGA NPs mixture (1:1), and ALA PLGA NPs were obtained on a DSC meter (Q2000; TA Instruments, New Castle, DE, USA). Samples $(\sim 2 \mathrm{mg}$ ) were sealed in aluminum pans and heated in an inert atmosphere of nitrogen. DSC curves were obtained between $15^{\circ} \mathrm{C}$ and $200^{\circ} \mathrm{C}$ with a heat rate of $10^{\circ} \mathrm{C} /$ minute.

\section{In vitro tests of ALA PLGA NPs \\ Cell line}

A431 cells derived from human epidermoid SCC (obtained from Shanghai Cell Bank, Shanghai, People's Republic of China) were routinely maintained in Roswell Park Memorial Institute (RPMI) 1640 medium supplemented with 10\% fetal bovine serum, penicillin (100 IU mL $\left.\mathrm{mL}^{-1}\right)$, streptomycin $\left(100 \mu \mathrm{g} \mathrm{mL}^{-1}\right)$, and $2 \mathrm{mM} \mathrm{L-glutamine.}$

\section{Observation of ALA PLGA NPs uptake using transmission electron microscopy}

A431 cells were incubated with serum-free medium (SFM) containing $2.7 \mathrm{mg} / \mathrm{mL}$ ALA PLGA NPs $(0.1 \mathrm{mM}$ ALA equivalent) or $0.1 \mathrm{mM} \mathrm{ALA}$ at $37^{\circ} \mathrm{C}$ for 4 hours. After being rinsed three times with cold PBS, cells were fixed in $2 \%$ glutaraldehyde for 2 hours and examined under transmission electron microscopy (CM-120; Philips).

\section{PpIX fluorescence kinetics measurement}

Fresh cells $\left(1 \times 10^{5}\right.$ cells/well $)$ were seeded in 96 -well black wall plates (Corning Inc, Corning, NY, USA) and cultured routinely for 24 hours. Attached cells were washed twice with SFM and grown in $100 \mu \mathrm{L}$ SFM containing $0 \mathrm{mM}$, $0.1 \mathrm{mM}$, and $1 \mathrm{mM}$ ALA, and ALA PLGA NPs $(0.1 \mathrm{mM}$ ALA equivalent), respectively. At predetermined time points, between 1 hour and 24 hours, the PpIX fluorescence was determined using a microplate reader (Synergy 2 multimode microplate reader; BioTek, Winooski, VT, USA). The fluorescence excitation wavelength was set at $400 \mathrm{~nm}$ and the emission wavelength at $635 \mathrm{~nm}$. Data were corrected for autofluorescence background.

\section{Comparison of ALA- and ALA PLGA NPs-mediated photocytotoxicity on A43 I cells}

Fresh cells $\left(1.0 \times 10^{4}\right.$ cells/well $)$ were seeded in 96 -well black wall plates and incubated for 24 hours. After being washed 
twice with SFM, the cells were incubated in $100 \mu \mathrm{L}$ SFM containing $0.1 \mathrm{mM}$ ALA, $1 \mathrm{mM}$ ALA, and ALA PLGA NPs ( $0.1 \mathrm{mM}$ ALA equivalent) for 6 hours or 24 hours. Then cells were irradiated by a helium-neon laser ( $632.8 \mathrm{~nm}$; Shanghai Laser Research Institute, Shanghai, People's Republic of China) at a power density of $8.6 \mathrm{~mW} / \mathrm{cm}^{2}$ and energy density of $8 \mathrm{~J} / \mathrm{cm}^{2}$. Following irradiation, the cells were incubated in fresh complete medium for another 24 hours before being subjected to MTT assay.

\section{Statistical analysis}

All experiments were repeated at least three times. The SPSS 13.0 software (IBM Corporation, Armonk, NY, USA) was used to perform statistical tests. Two-way anaysis of variance (ANOVA) was used to compare the time course of PpIX generation by ALA PLGA NPs and $0.1 \mathrm{mM}$ ALA. Independent $t$-test was used to compare the PpIX fluorescence at a selected time point and also the cell survival of different groups. $P<0.05$ was considered statistically significant.

\section{Results}

\section{Characteristics of ALA PLGA NPs}

Particle size analysis showed that the number-weighted mean diameter of ALA PLGA NPs was $65.6 \mathrm{~nm} \pm 26 \mathrm{~nm}$. The $\mathrm{D}_{10}$, $\mathrm{D}_{50}$, and $\mathrm{D}_{90}$ of ALA PLGA NPs was $46.1 \mathrm{~nm}, 61.2 \mathrm{~nm}$, and $84 \mathrm{~nm}$, respectively. The PI, EE and LC of ALA PLGA NPs was $0.62,65.8 \% \pm 7.2 \%$ and $0.62 \% \pm 0.27 \%$, respectively.

Scanning electron microscopic examination showed that ALA PLGA NPs were spherical with smooth surfaces and good dispersion (Figure 2A). The diameters of ALA PLGA NPs ranged from $20 \mathrm{~nm}$ to $500 \mathrm{~nm}$ (Figure 2B), which were consistent with that obtained by the particle size analyzer.

Thermograms showed that pure ALA had a melting endothermic peak at $158.04^{\circ} \mathrm{C}$ (Figure 3A). There was no peak observed at this temperature for empty PLGA NPs (Figure 3B). ALA and PLGA NPs mixture showed a similar melting endothermic peak at $157.09^{\circ} \mathrm{C}$, but the peak height
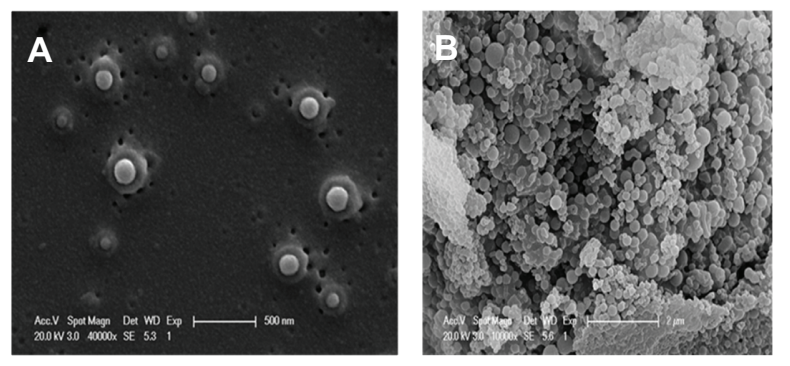

Figure 2 Morphology of ALA PLGA NPs (5-aminolevulinic acid poly[lactic-coglycolic acid] nanoparticles). (A) 40,000x; (B) 10,000x. was lower (Figure 3C). There was no peak observed for ALA PLGA NPs (Figure 3D). This was due to the amorphous state of ALA when dispersed in the NPs.

\section{Uptake of ALA PLGA NPs in A43 I cells}

Transmission electron microscopic examination showed that after 4 hours of incubation with ALA, there was no apparent change in cell structures (Figure 4A and B). For the ALA PLGA NPs group, a large number of spherical NPs could be seen in A431 cells. Inside cells, most NPs were intact, while a small number of them became irregular in shape (Figure 4C and D). In addition, ALA PLGA NPs were mainly gathered in the cytoplasm, while there were no ALA PLGA NPs in the nucleus (Figure 4E and F).

\section{Kinetics of PpIX fluorescence}

After incubating with $1 \mathrm{mM}$ ALA, PpIX fluorescence intensity in A431 cells increased with time. There was no significant PpIX formation after incubating with $0.1 \mathrm{mM}$ ALA, but there was noticeable PpIX formation after incubating with ALA PLGA NPs containing 0.1 mM ALA. The PpIX fluorescence generated by ALA PLGA NPs was higher than that generated by $0.1 \mathrm{mM}$ ALA $(P=0.000)$. Interestingly, during early hours (ie, 1-8 hours), the PpIX fluorescence generated by ALA PLGA NPs was even higher than that generated by $1 \mathrm{mMALA}$. For instance, after 6 hours of incubation, the PpIX fluorescence generated by ALA PLGA NPs was higher than that generated by $0.1 \mathrm{mM}$ $\operatorname{ALA}(P=0.002)$ and $1 \mathrm{mM} \mathrm{ALA}(P=0.036)$ (Figure 5).

\section{ALA- and ALA PLGA NPs-mediated photocytotoxicity effect}

MTT assay showed that ALA and ALA PLGA NPs alone did not affect cell viability (data not shown). ALA- and ALA PLGA NPs-mediated photocytotoxicity could be affected by the incubation time. For 6 hours of incubation, the photocytotoxicity of ALA PLGA NPs was much higher than that of $0.1 \mathrm{mMALA}(P=0.000)$ but slightly lower than that of $1 \mathrm{mM}$ $\operatorname{ALA}(P=0.029)$ (Figure 6A). For 24 hours of incubation, the photocytotoxicity of ALA PLGA NPs was much lower than that of $1 \mathrm{mM}$ ALA $(P=0.002)$ but much higher than that of $0.1 \mathrm{mM}$ ALA $(P=0.006)$ (Figure 6B). These results correlated well with the kinetics of PpIX formation.

\section{Discussion}

ALA is a prodrug of porphyrins and widely used in skin PDT around the world, but the quantification of ALA is still challenging since it is a very weak chromophore. A liquidchromatographic method used for determining ALA in 

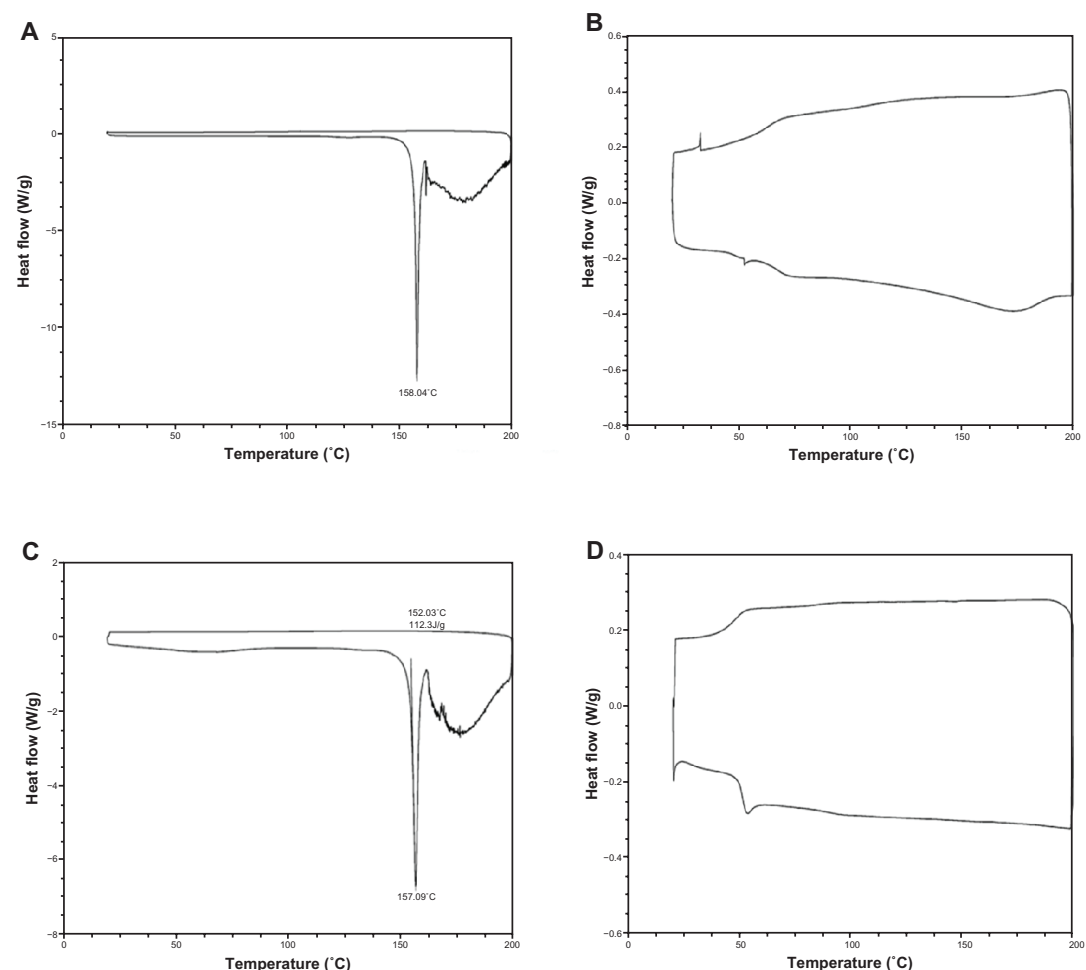

Figure 3 Differential scanning calorimetry thermograms of ALA (A), PLGA NPs (B), ALA and PLGA NPs mixture (C), and ALA PLGA NPs (D). Abbreviatons: ALA, 5-aminolevulinic acid; PLGA, poly(lactic-co-glycolic acid); NPs, nanoparticles.

biological samples was first reported by Tomokuni et a ${ }^{17}$ in 1987 and later optimized by Oishi el at ${ }^{18}$ in 1996 . However heating at $100^{\circ} \mathrm{C}$ was required in their method, which can lead to the degradation of ALA. ${ }^{19}$ Namjoshi et al developed
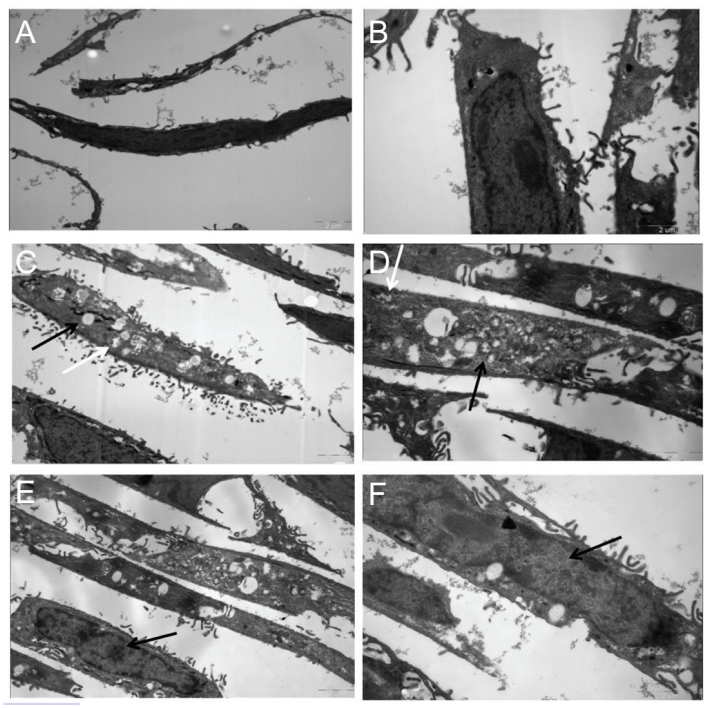

Figure 4 Transmission electron microscope photographs of A43I cells incubated with ALA (A) 7,400× and (B) 13,500× or with ALA PLGA NPs (5-aminolevulinic acid poly[lactic-co-glycolic acid] nanoparticles). (C and E) 7,400× and (D and F) 13,500×. Note: The white arrows indicate the irregular nanoparticles, and the black arrows indicate the intact nanoparticles and nucleus.

Abbrevations: ALA, 5-aminolevulinic acid; PLGA, poly(lactic-co-glycolic acid); NPs, nanoparticles. a derivatization reaction that can be carried out at room temperature using fluorescamine, which reacts readily and instantaneously with ALA at room temperature. ${ }^{15}$ After the reaction is complete, the residual fluorescamine is rapidly converted to nonfluorescent products. This method provides a reliable, simple, and quick alternative for ALA determination.

Topical ALA-PDT is a useful modality for several skin and mucosal diseases; $;^{2-4}$ however, the insufficient local bioavailability, instability in physiological environment, and the poor diffusion of ALA across cell membranes can limit the

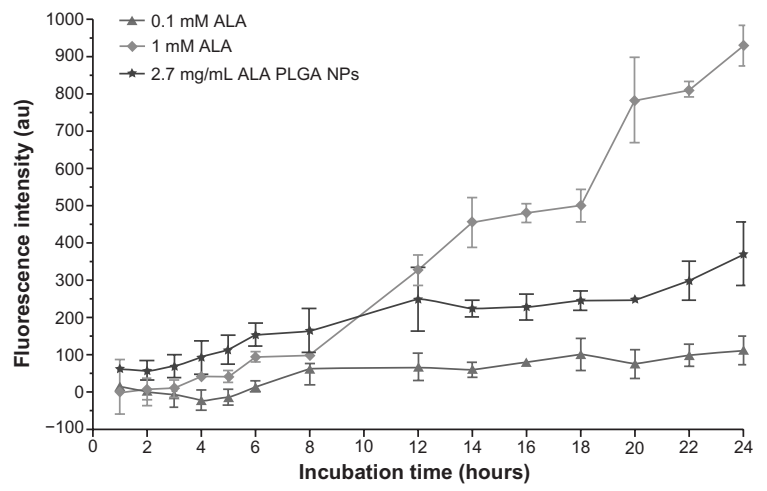

Figure 5 Protoporphyrin IX accumulation in A43I cells.

Notes: Data were corrected for autofluorescence and presented as median \pm standard deviation; $(n=3)$.

Abbrevations: ALA, 5-aminolevulinic acid; PLGA, poly(lactic-co-glycolic acid); NPs, nanoparticles. 

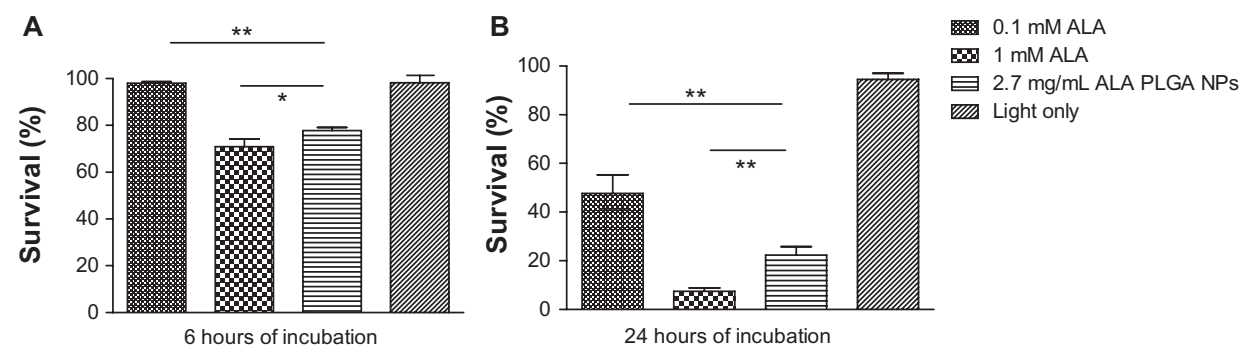

Figure 6 MTT assay of photodynamic therapy effect on A43I cells.

Notes: Cells were incubated with ALA or ALA PLGA for 6 hours (A) or 24 hours (B). Data were presented as mean \pm standard deviation from at least three independent experiments. $* P<0.05$, ** $P<0.01$.

Abbreviatons: ALA, 5-aminolevulinic acid ; MTT, 3-(4,5)-dimethylthiahiazo(-z-yl)-3,5-di-phenytetrazoliumromide.

effectiveness of ALA-PDT. Overcoming these limitations can improve the efficacy of ALA-PDT in the treatment of skin SCC. Several methods have been suggested for improving ALA transdermal delivery, which include microneedles, patch-based systems, microemulsions, iontophoresis, and laser. ${ }^{7,20-22}$ Extensive research has also been carried out to develop NP drug delivery systems for ALA, ${ }^{8-11}$ but PLGA NPs have not been tested for ALA delivery before.

PLGA has been used as a nanocarrier for many bioactive molecules (eg, drugs, peptides, proteins, DNA, and oligonucleotides) owing to its low toxicity, good biocompatibility, and FDA approval status. ${ }^{12}$ In this study, we used a modified double emulsion solvent evaporation method to entrap ALA in PLGA NPs. Our results showed that ALA PLGA NPs had a relatively good particle size distribution $(65.6 \mathrm{~nm} \pm 26 \mathrm{~nm})$ and high EE $(65.8 \% \pm 7.2 \%)$, although small hydrophilic molecules are not considered an ideal candidate for PLGA NPs.

The DSC thermograms suggested that ALA was in an amorphous phase in the NPs, which might contribute to enhancing the efficacy of ALA-PDT. The stability of ALA in aqueous solutions can be affected by $\mathrm{pH}$, concentration, temperature, and degree of oxygenation of the solution. Among them, $\mathrm{pH}$ is particularly important. ALA dimerizes readily under alkaline conditions and forms dihydropyrazines, which can be further oxidized to pyrazines. The zwitterion is the major species of ALA present in the $\mathrm{pH}$ range between 5 and 7.5. At pH 5.0, approximately $10 \%$ of the ALA molecules are cations, whereas at $\mathrm{pH} 7.3$, approximately $10 \%$ of the ALA molecules are anions. The anion, a species with a deprotonated amino group, is able to react with the ketone group of nearby molecules to yield the cyclic dihydropyrazine. For the condensation to occur, the amino group of ALA should be deprotonated. This explains the strong $\mathrm{pH}$ dependence of the reaction since the concentration of the anion increases with $\mathrm{pH}$. ALA solutions are more stable at low $\mathrm{pH}$ values, where the anion almost does not exist. Therefore, the preparation of ALA PLGA NPs should be carried out at low $\mathrm{pH}$.

It should be noted that the step of extracting ALA from DCM solution of polymer might result in a loss of ALA and consequently affect the measurement of encapsulation and loading efficiency. The primary emulsion was transferred into the external aqueous phase consisting of $1 \%$ polyvinyl alcohol in PBS (pH 5.0). The fraction of polyvinyl alcohol may be associated with the nanocapsules (despite repeated washing) due to the formation of an interconnected polyvinyl alcohol/polymer network at the nanocapsule interface. The residual polyvinyl alcohol could alter the biopharmaceutical features of nanoparticles by decreasing the cellular uptake..$^{23}$ In the present study, we also tried utilizing different concentrations of polyvinyl alcohol. Since the increase of polyvinyl alcohol concentration did not improve the particle size of PLGA NPs (data not shown), $1 \%$ polyvinyl alcohol was used. In addition, we also tested the high pressure homogenization for ALA PLGA NPs preparation. The result showed that the mean particle size of NPs was less than $50 \mathrm{~nm}$, but the EE was low (data not shown). This might be due to the tremendous destructive power of high pressure homogenization. Nonetheless, the properties of PLGA NPs might be improved through covalent attachment to polyethylene glycol polymer chains (ie, PEGylation). ${ }^{24}$

The concentration of $2.7 \mathrm{mg} / \mathrm{mL}$ of ALA PLGA NPs (equivalent to $0.1 \mathrm{mM} \mathrm{ALA}$ ) was selected in this study. This was because a higher concentration $(>10 \mathrm{mg} / \mathrm{mL})$ of ALA PLGA NPs suspension would be prone to coagulation. The LC of ALA PLGA NPs prepared in this study was low $(0.62 \% \pm 0.27 \%)$. If the ALA LC of PLGA NPs could be further improved, the same amounts of PLGA NPs would be able to load more ALA.

A large number of spherical ALA PLGA NPs were located in the cytoplasm of A431 cells (see Figure 4). After 4 hours of incubation, the majority of ALA PLGA NPs in 
the cytoplasm were spherical, but a small portion of ALA PLGA NPs presented as irregular shapes, indicating that the degradation might have begun. We inferred that whole ALA PLGA NPs were taken up by cells first, and then ALA was released following the degradation of ALA PLGA NPs in the cytoplasm. PLGA NPs delivered ALA directly into the cytoplasm and consequently PpIX was generated in the cytoplasm and mitochondria. The stability of ALA might be improved and the consumption of extracellular ALA reduced due to the encapsulation, which can generate more PpIX.

ALA PLGA NPs and ALA-mediated PpIX fluorescence in A431 cells increased with the incubation time (see Figure 5). This result is consistent with other studies. ${ }^{25,26}$ The PpIX production and photocytotoxicity induced by ALA PLGA NPs containing $0.1 \mathrm{mM}$ ALA were higher than that produced by $0.1 \mathrm{mM}$ free ALA (see Figure 6). This is possibly because free ALA might be directly dissolved in the extracellular compartment and decomposes, whereas PLGA NPs could protect ALA from such degradation.

Interestingly, PpIX fluorescence kinetics results showed that A431 cells incubated with ALA PLGA NPs generated higher levels of PpIX than those incubated with $1 \mathrm{mM}$ free ALA during the first 8 hours of incubation. This may also be owing to the different cellular uptake routes of ALA PLGA NPs versus the free ALA. Diffusion of free ALA through cell membranes is limited because of its polarity. Free ALA is mainly taken up intracellularly by an active, energyconsuming mechanism via $b$-amino acid and $\gamma$-amino butyric acid carriers. ${ }^{27}$ The transport rate is restricted since a large amount of ALA in the extracellular environment continues to degrade. However, ALA PLGA NPs enter cells principally by endocytosis, especially via dynamin-dependent/ clathrin-mediated endocytosis pathway. ${ }^{28}$ In addition, these NPs might enter the cell directly through discontinuities in the plasma membrane, either preexisting or induced by the NPs through direct permeabilization of the cell surface. These mechanisms might be the main reason for the enhancement of cellular ALA uptake and PpIX formation. ${ }^{29}$

It was noted that the PpIX fluorescence of A431 cells incubated with free ALA was not increased quickly during the first 4 hours of incubation. This might be because of the potential drug efflux transporters in A431 cell membranes, such as P-glycoprotein and multidrug resistance relatedprotein. These drug efflux transporters could pump ALA and PpIX out of cells. ${ }^{27}$ In the early stage of ALA uptake, the amount of intracellular ALA was limited, coupled with exocytosis by drug efflux transporters at the same time, resulting in ALA and/or PpIX concentrations insufficient to overload the heme synthesis pathway. It has been reported that PLGA NPs could also inhibit theses drug efflux transporters. ${ }^{30}$

The trend of PpIX formation over time in A431 cells is different than the bell-shape curve often seen in vivo (see Figure 5). In the in vivo case, the tumor growth is often accompanied by a complete vascular system. The body can supply a large amount of iron to convert PpIX to heme. So following a 2-6 hour application of ALA, fluorescence intensity reaches its peak and begins to decrease in tumor or normal skin if extending into the occlusion time. ${ }^{25}$ In the current clinical practice, the best occlusion/incubation time for ALA/PpIX is approximately 6 hours. Therefore, PpIX levels generated in the first 6 hours are of vital importance. It should be pointed out that various types of NPs might be used to deliver PpIX as well. ${ }^{24}$ Nonetheless, the roles of nanocarriers in ALA delivery and PpIX formation deserve further in vivo investigation.

The inhibition of cell proliferation was consistent with PpIX fluorescence kinetics, indicating that ALA PLGA NPs work mainly through increasing the amount and rate of PpIX formation to enhance the efficiency of the photodynamic reaction. As mentioned earlier, in clinical practice, the irradiation is often carried out following the topical application of ALA for 6 hours. Therefore, the results of the 6-hour incubation may have a greater clinical relevance. At 6 hours, the killing rate of PDT mediated with $2.7 \mathrm{mg} / \mathrm{mL}$ ALA PLGA NPs (0.1 mM ALA equivalent) was slightly lower than that of $1 \mathrm{mM}$ free ALA but much higher than that of $0.1 \mathrm{mM}$ free ALA, suggesting that PLGA NPs increased the killing effects of ALA-PDT. Apparently, to achieve the same therapeutic effect, ALA PLGA NPs PDT requires lower doses of ALA. This might reduce the side effects and cost.

\section{Conclusion}

The present study demonstrates that a modified double emulsion solvent evaporation method is a suitable technique for the preparation of PLGA NPs containing prodrug ALA for treating SCC cells. ALA PLGA NPs can enhance in vitro ALA-PDT efficiency via producing more PpIX at a low ALA concentration in SCC cells. The combination of ALA and PLGA NPs might provide a promising strategy for delivering ALA for topical PDT. The roles of nanocarriers in transdermal ALA delivery and PpIX formation deserve further in vivo investigation.

\section{Acknowledgments}

This work was supported by grants from the National Natural Science Foundation (81272990), the Shanghai Natural Science Foundation (11ZR1432800), and the Key Project of 
Shanghai Municipal Health Bureau (20124034). The authors would like to thank Li Yang and Yazhen Wang of the National Pharmaceutical Engineering Research Center for assisting in nanoparticle preparation.

\section{Disclosure}

The authors report no conflicts of interest in this work.

\section{References}

1. Huang Z, Xu H, Meyers AD, et al. Photodynamic therapy for treatment of solid tumors - potential and technical challenges. Technol Cancer Res Treat. 2008;7(4):309-320.

2. Wang XL, Wang HW, Wang HS, Xu SZ, Liao KH, Hillemanns P. Topical 5-aminolaevulinic acid-photodynamic therapy for the treatment of urethral condylomata acuminata. Br J Dermatol. 2004;151(4):880-885.

3. Babilas P, Landthaler M, Szeimies RM. Photodynamic therapy in dermatology. Eur J Dermatol. 2006;16(4):340-348.

4. Wang XL, Wang HW, Guo MX, Xu SZ. Treatment of skin cancer and pre-cancer using topical ALA-PDT - a single hospital experience. Photodiagnosis Photodyn Ther. 2008;5(2):127-133.

5. Ishizuka M, Abe F, Sano Y, et al. Novel development of 5-aminolevurinic acid (ALA) in cancer diagnoses and therapy. Int Immunopharmacol. 2011;11(3):358-365.

6. Wang H, Li J, Lv T, Tu Q, Huang Z, Wang X. Therapeutic and immune effects of 5-aminolevulinic acid photodynamic therapy on UVBinduced squamous cell carcinomas in hairless mice. Exp Dermatol. 2013;22(5):362-363.

7. Araújo LM, Thomazine JA, Lopez RF. Development of microemulsions to topically deliver 5-aminolevulinic acid in photodynamic therapy. Eur J Pharm Biopharm. 2010;75(1):48-55.

8. Ding H, Sumer BD, Kessinger CW, et al. Nanoscopic micelle delivery improves the photophysical properties and efficacy of photodynamic therapy of protoporphyrin IX. $J$ Control Release. 2011;151(3):271-277.

9. Yang SJ, Shieh MJ, Lin FH, et al. Colorectal cancer cell detection by 5-aminolaevulinic acid-loaded chitosan nano-particles. Cancer Lett. 2009;273(2):210-220.

10. Ito S, Miyoshi N, Degraff WG, Nagashima K, Kirschenbaum LJ, Riesz P. Enhancement of 5-aminolevulinic acid-induced oxidative stress on two cancer cell lines by gold nanoparticles. Free Radic Res. 2009;43(12):1214-1224.

11. Oh EK, Jin SE, Kim JK, Park JS, Park Y, Kim CK. Retained topical delivery of 5-aminolevulinic acid using cationic ultradeformable liposomes for photodynamic therapy. Eur J Pharm Sci. 2011;44(1-2):149-157.

12. Lü JM, Wang X, Marin-Muller C, et al. Current advances in research and clinical applications of PLGA-based nanotechnology. Expert Rev Mol Diagn. 2009;9(4):325-341.

13. Allison RR, Mota HC, Bagnato VS, Sibata CH. Bio-nanotechnology and photodynamic therapy - state of the art review. Photodiagnosis Photodyn Ther. 2008;5(1):19-28.

14. Korbelik M, Madiyalakan R, Woo T, Haddadi A. Antitumor efficacy of photodynamic therapy using novel nanoformulations of hypocrellin photosensitizer SL052. Photochem Photobiol. 2012;88(1): 188-193.

International Journal of Nanomedicine

\section{Publish your work in this journal}

The International Journal of Nanomedicine is an international, peerreviewed journal focusing on the application of nanotechnology in diagnostics, therapeutics, and drug delivery systems throughout the biomedical field. This journal is indexed on PubMed Central, MedLine, CAS, SciSearch ${ }^{\circledR}$, Current Contents ${ }^{\circledR} /$ Clinical Medicine,
15. Namjoshi S, Caccetta R, Edwards J, Benson HA. Liquid chromatography assay for 5-aminolevulinic acid: application to in vitro assessment of skin penetration via dermaportation. J Chromatogr B Analyt Technol Biomed Life Sci. 2007;852(1-2):49-55.

16. Mondalek FG, Lawrence BJ, Kropp BP, et al. The incorporation of poly(lactic-co-glycolic) acid nanoparticles into porcine small intestinal submucosa biomaterials. Biomaterials. 2008;29(9):1159-1166.

17. Tomokuni K, Ichiba M, Hirai Y, Hasegawa T. Optimized liquidchromatographic method for fluorometric determination of urinary delta-aminolevulinic acid in workers exposed to lead. Clin Chem. 1987;33(9):1665-1667.

18. Oishi H, Nomiyama H, Nomiyama K, Tomokuni K. Fluorometric HPLC determination of delta-aminolevulinic acid (ALA) in the plasma and urine of lead workers: biological indicators of lead exposure. $J$ Anal Toxicol. 1996;20(2):106-110.

19. Giuntini F, Bourré L, Macrobert AJ, Wilson M, Eggleston IM. Quantitative determination of 5-aminolaevulinic acid and its esters in cell lysates by HPLC-fluorescence. J Chromatogr B Analyt Technol Biomed Life Sci. 2008;875(2):562-566.

20. Donnelly RF, Morrow DI, McCarron PA, et al. Microneedle-mediated intradermal delivery of 5 -aminolevulinic acid: potential for enhanced topical photodynamic therapy. J Control Release. 2008;129(3): 154-162.

21. Morrow DI, McCarron PA, Woolfson AD, et al. Novel patch-based systems for the localised delivery of ALA-esters. $J$ Photochem Photobiol B. 2010;101(1):59-69.

22. Merclin N, Bender J, Sparr E, Guy RH, Ehrsson H, Engström S. Transdermal delivery from a lipid sponge phase - iontophoretic and passive transport in vitro of 5-aminolevulinic acid and its methyl ester. J Control Release. 2004;100(2):191-198.

23. Sahoo SK, Panyam J, Prabha S, Labhasetwar V. Residual polyvinyl alcohol associated with poly (D,L-lactide-co-glycolide) nanoparticles affects their physical properties and cellular uptake. $J$ Control Release. 2002;82(1):105-114.

24. Paszko E, Ehrhardt C, Senge MO, Kelleher DP, Reynolds JV. Nanodrug applications in photodynamic therapy. Photodiagnosis Photodyn Ther. 2011;8(1):14-29.

25. Lang K, Bolsen K, Stahl W, et al. The 5-aminolevulinic acid-induced porphyrin biosynthesis in benign and malignant cells of the skin. J Photochem Photobiol B. 2001;65(1):29-34.

26. Chung CW, Kim CH, Choi KH, et al. Effect of surfactant on 5-aminolevulinic acid uptake and PpIX generation in human cholangiocarcinoma cell. Eur J Pharm Biopharm. 2012;80(2):453-458.

27. Rud E, Gederaas O, Høgset A, Berg K. 5-aminolevulinic acid, but not 5-aminolevulinic acid esters, is transported into adenocarcinoma cells by system BETA transporters. Photochem Photobiol. 2000; 71(5):640-647.

28. Fernando LP, Kandel PK, Yu J, McNeill J, Ackroyd PC, Christensen KA. Mechanism of cellular uptake of highly fluorescent conjugated polymer nanoparticles. Biomacromolecules. 2010;11(10):2675-2682.

29. Smith PJ, Giroud M, Wiggins HL, et al. Cellular entry of nanoparticles via serum sensitive clathrin-mediated endocytosis, and plasma membrane permeabilization. Int J Nanomedicine. 2012;7:2045-2055.

30. Wang Y, Guo M, Lu Y, et al. Alpha-tocopheryl polyethylene glycol succinate-emulsified poly(lactic-co-glycolic acid) nanoparticles for reversal of multidrug resistance in vitro. Nanotechnology. 2012;23(49):495103.

\section{Dovepress}

Journal Citation Reports/Science Edition, EMBase, Scopus and the Elsevier Bibliographic databases. The manuscript management system is completely online and includes a very quick and fair peer-review system, which is all easy to use. Visit http://www.dovepress.com/ testimonials.php to read real quotes from published authors. 